\title{
GEOSPATIAL MODELLING APPROACH FOR 3D URBAN DENSIFICATION DEVELOPMENTS
}

\author{
O. Koziatek ${ }^{\text {a }}$, S. Dragićević ${ }^{a^{*}, S . ~ L i ~}{ }^{b}$ \\ ${ }^{a}$ Dept. of Geography, Simon Fraser University, Burnaby, Canada - (oka8, suzanad)@ sfu.ca \\ ${ }^{\mathrm{b}}$ Dept. of Civil Engineering, Ryerson University, Toronto, Canada - snli@ reyrson.ca
}

Commission II, WG II/5

KEY WORDS: geospatial modelling, 3D GIS, urban densification, urban planning, vertical growth

\begin{abstract}
:
With growing populations, economic pressures, and the need for sustainable practices, many urban regions are rapidly densifying developments in the vertical built dimension with mid- and high-rise buildings. The location of these buildings can be projected based on key factors that are attractive to urban planners, developers, and potential buyers. Current research in this area includes various modelling approaches, such as cellular automata and agent-based modelling, but the results are mostly linked to raster grids as the smallest spatial units that operate in two spatial dimensions. Therefore, the objective of this research is to develop a geospatial model that operates on irregular spatial tessellations to model mid- and high-rise buildings in three spatial dimensions (3D). The proposed model is based on the integration of GIS, fuzzy multi-criteria evaluation (MCE), and 3D GIS-based procedural modelling. Part of the City of Surrey, within the Metro Vancouver Region, Canada, has been used to present the simulations of the generated 3D building objects. The proposed 3D modelling approach was developed using ESRI's CityEngine software and the Computer Generated Architecture (CGA) language.
\end{abstract}

\section{INTRODUCTION}

The global population is increasing rapidly and placing pressure on urban development. Cities are challenged by opposing economic and sustainable development demands. Urban growth sometimes identified as urban sprawl, means that development is occurring more in the horizontal direction. Sustainability concerns pressure urban planners and developers to consider alternative urban growth strategies, such as vertical and compact development, to augment the urban population densities and to reduce the adverse environmental impact of urban sprawl.

Current literature indicates that three dimensional (3D) vertical urban growth models have been developed using spatial statistics and remote sensing approaches but with little temporal consideration. Remote sensing imagery and recently LiDAR data have been used mainly for urban vertical change detection (Wang, 2013). Spatial distribution of high-rise buildings were modelled using multi-nominal logit (Frenkel, 2004), threetiered decision making approach (Tamošaitienė et al., 2013), and descriptive statistics coupled with regression analysis and principal component analysis (Salvatie et al., 2013). Cellular automata (CA) geosimulation approaches have been extensively used to model space-time dynamics of urban sprawl processes in the last two decades (Batty, 1999; White and Engelen, 1993) but the built environment was presented in two spatial dimensions. Benguiui and Czamanski (2008) and Li et al. (2014) proposed CA models to address urban densification and vertical growth. However, these approaches resulted in low resolution regular grid distributions, not representative of the shape and size of typical irregular cadastral lots. Although advancements have been made on irregularly shaped grids in urban CA modelling (Stevens et al., 2007), more work is necessary to improve compatibility and ease of these limitations. Cadastral lots are fundamental units in planning; therefore modelling vertical urban growth would be more useful if the shape and size of these units are represented.

The main goal of this research study is to propose a geospatial modelling approach to represent the urban densification process in $3 \mathrm{D}$ by generating urban development in the form of mid- and high-rise buildings. Integration of geographic information science (GIS), multi-criteria evaluation (MCE), and procedural modelling have been used to develop the model. This proposed approach addresses the concerns surrounding low resolution regular grid models by projecting scenarios of 3D building developments on irregular cadastral lots.

\section{METHODS}

\subsection{D growth parameters}

Factors affecting urban densification and vertical urban growth have been studied by Burton (2002), Frenkel (2007), Mubareka et al. (2011), Sani Roychansyah et al. (2005), and Turskis et al. (2006). Urban densification, often named urban compactness, occurs in areas of higher population density and is often correlated to locations of mid- and high-rise buildings. The identified key factors contributing to vertical urban growth are related to population demographics and growth, economic opportunities, availability and accessibility to transportation networks, social and environmental services and activities, and land use designations. Main factors used in this study are population density, land use, distance to local services and amenities, transportation, job centres, and community and outdoor activities.

\footnotetext{
*Corresponding Author
} 


\subsection{Study site and data sets}

The Metro Vancouver Region in Canada has a growing population with development constrained by water, mountains, agricultural land reserve, and forests. The City of Surrey was selected as a study area because it is characterized as one of the fastest growing cities in Canada and the Metro Vancouver Region (City of Surrey, 2016), and offers accessible geospatial data. Particularly, this study focused on the Guilford Town Centre as sub-study area of the city of Surrey (Figure 1a). GIS data sets such as city lots, buildings, land use designation, parks, schools, transportation networks, hazardous lands, and city centres were obtained from the City of Surrey's Open Data website (City of Surrey, 2016b). Population data was obtained from census records for year 2011 (Statistics Canada, 2011) at the dissemination area level. The raster GIS data layers used for the land evaluation analysis were at $10 \mathrm{~m}$ spatial resolution.

\subsection{Modelling approach}

The proposed geospatial modelling approach integrates GISbased fuzzy multi-criteria evaluation (MCE) method and procedural modelling, which is accomplished in two steps. The first step is related to the evaluation of suitable locations for vertical urban growth. The MCE methods are well known decision-making approaches that have been used for different geographical applications in land site selection, agricultural land preservation, and urban and regional planning (Voogd, 1983; Carver, 1991; Malczewski, 2004). The fuzzy MCE method was used to provide suitability scores ranging from 0 to 1 where 0 indicates unsuitable and 1 indicates highly suitable locations for a vertical development. The MCE criteria were selected from the factors that were identified as the main contributors to vertical urban growth such as importance of recreation and green space, economic opportunity, distance to transportation, services and amenities, land use type, and population density. The MCE criteria were represented by fuzzy suitability functions to include planning strategies described in the City of Surrey by-laws (City of Surrey, 2014). The ESRI ArcGIS 10.2 software (ESRI, 2013) was used to combine multiple GIS data layers and implement the MCE method. Once the locations of potential suitable sites for vertical growth were identified, the results were utilized in the second stage consisting of a 3D GIS-based procedural model to generate building objects.

The procedural modelling approach creates 3D objects from the existing geometry based on the refinement rules (Parish and Müller, 2001). This geometry is extracted from existing vectorbased GIS data that represents irregular spatial tessellations, which creates 3D objects based on L-system grammar. The procedural rules encompass regional and urban building development polices and city-bylaws, including the size of land subdivisions and cadastral lots, building types, set-backs, and heights. This second step was operationalized by using ESRI's City Engine 2014.0 software (ESRI, 2014) as the 3D GIS-based procedural modelling environment, using Computer Graphic Architecture (CGA) shape grammar language in order to program rules. CGA rules are applied using architecture design and object transformation rules such as scale, rotate, translate, and add (Halatsch et al., 2008). The refinement rules were programmed to generate vertical urban growth based on the following hierarchy: 1) creating subdivisions of existing cadastral lots into smaller lots based on surface area parameter specifications; 2) assigning buildings' floor heights, setbacks, and colour to each land use designation using the land use reference script; 3) assigning land use designation to each cadastral lot determined by spatial alignment using the land use reference script; and 4) linking the cadastral lots to the obtained suitability scores and restricting growth to optimal locations.

\section{RESULTS}

The Guilford Town Centre, within the City of Surrey, is a wellknown shopping and expanding business district, was selected for the implementation of the developed geospatial modelling approach. The perimeter of this location is $1.3 \mathrm{~km}$ by $2 \mathrm{~km}$ and encompasses an approximate $2.6 \mathrm{~km}^{2}$ area (Figure 1a). The obtained suitability values from the GIS-based MCE methods in the first step of the modelling process are presented in Figure $1 b$, overlaying the values on the Google Earth 3D map. The values of highest suitability scores are in red, and the lowest in dark blue. The Google Earth 3D mid- and high- rise buildings reside within the suitable locations, which indicates that the MCE stage of the model has provided appropriate suitability values.
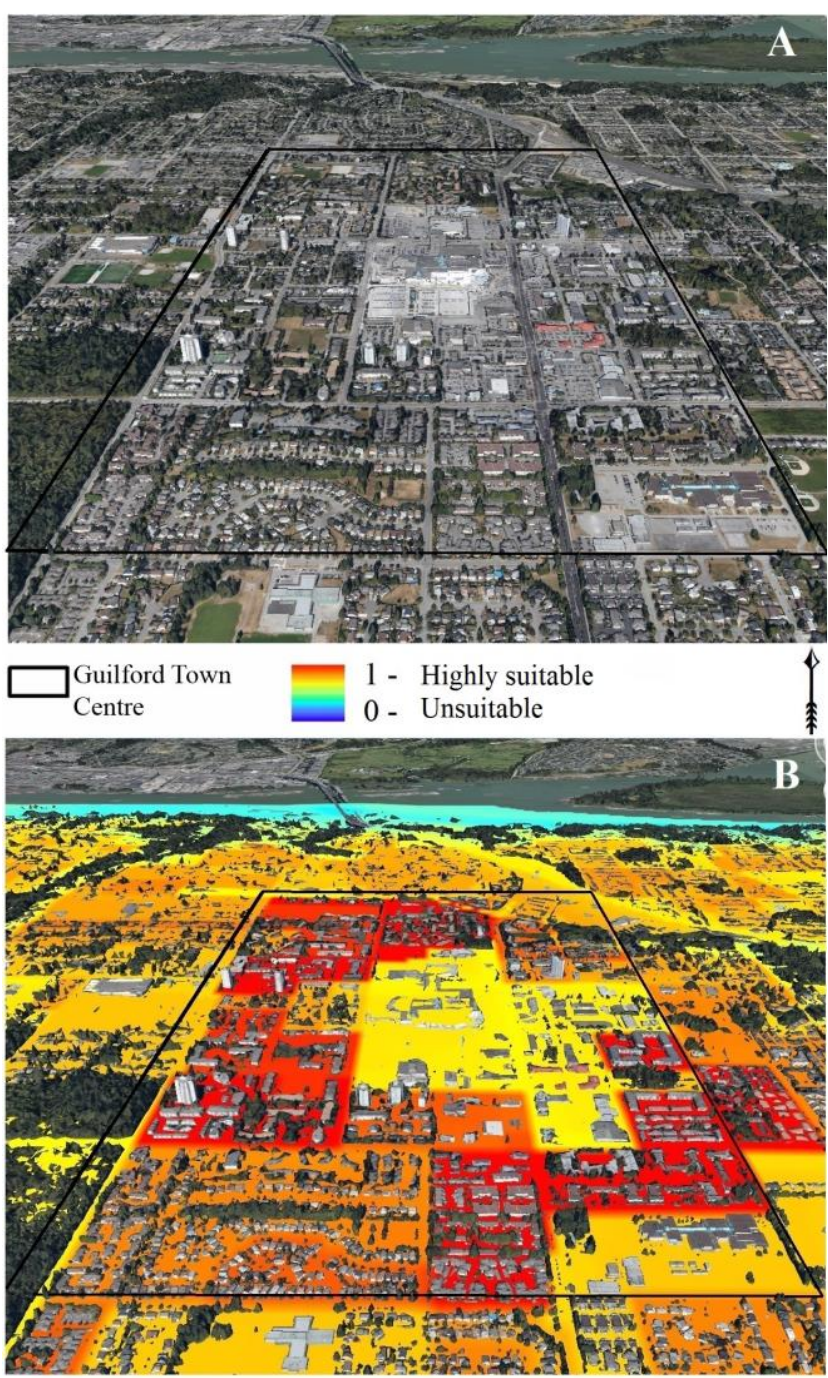

Figure 1. Guilford town centre, City of Surrey, Canada as (a) study area using Google Earth 3D building representation and (b) with obtained GIS-MCE map for vertical growth suitability

Stage two of the proposed geospatial model is related to the 3D GIS-based procedural modelling approach. The land use 
designations and exiting cadastral lot subdivisions from the City of Surrey (Figure 2) have been further subdivided for the vertical growth. The land use designations determine the type of buildings that can be generated on each new lot. Three scenarios have been designed to represent slow, moderate, and fast growth speeds for the 3D vertical urban developments to accommodate diverse population influx in the city. These growth scenarios were determined by using three different values for MCE suitability scores, which generated maps of varying building development land constrictions. The obtained modelling results are presented for slow (Figure 3a), moderate (Figure 3b) and fast growth (Figure 3c). The generated building objects in each scenario are colour-coded according to the land use designation of the developed lot.

The generated three growth scenarios are presented through a different perspective angle (Figure 4). The current city skylines with real extruded building footprint geometry are shown in dark grey (Figure 4a). The simulated development for slow (Figure 4b), moderate (Figure 4c), and fast growth (Figure 4d) are presented in designated building colours. The modelling results indicate the locations where the projected 3D growth occurred on the subdivided lots for each scenario. There is a noticeable increase in building densification surrounding the Guildford Town Centre, which is linked to the increased growth speed.

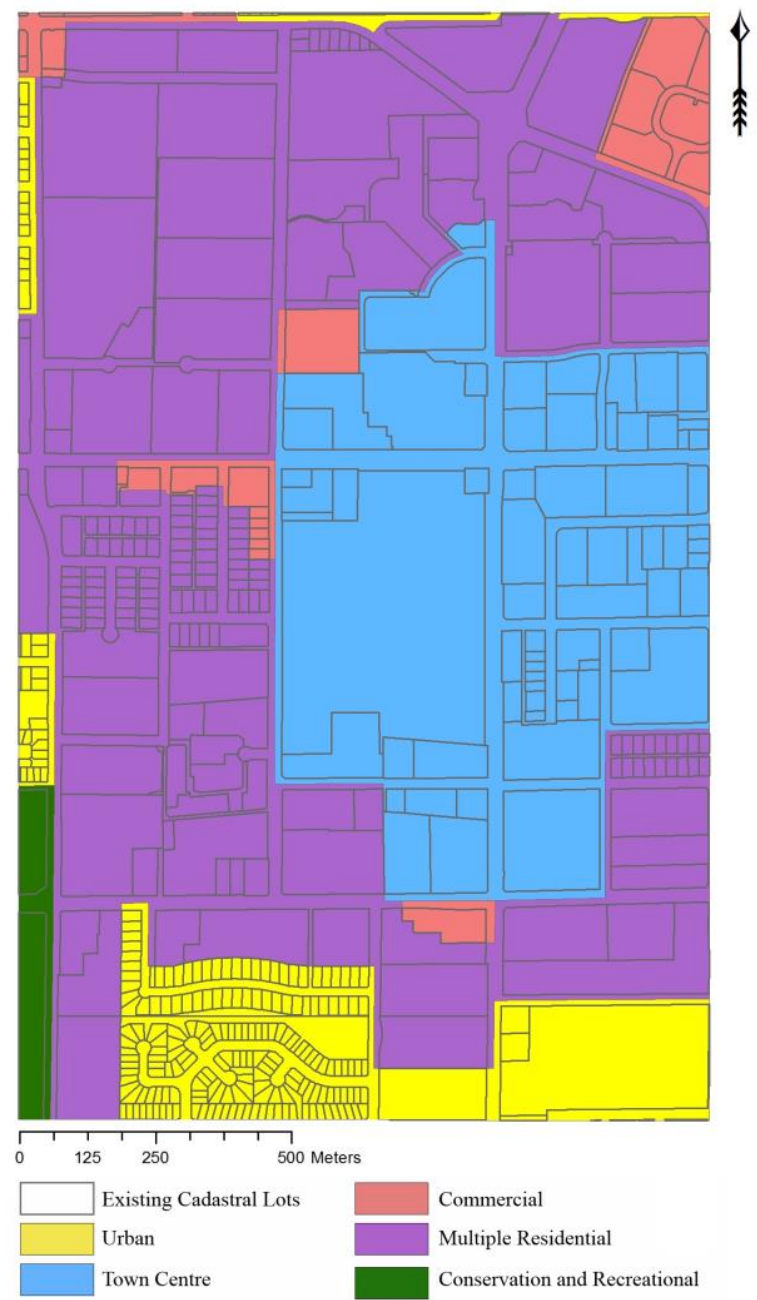

Figure 2. The City of Surrey land designations and existing cadastral subdivisions defining the building types

\section{CONCLUSION}

The developed geospatial vertical urban growth modelling approach utilizes the GIS-based fuzzy MCE method in combination with procedural modelling to project future urban densification. The model was implemented on cadastral lots of irregular shapes and sizes at high resolution, overcoming past limitations of raster based models. The three growth scenarios demonstrated a realistic progression of urban densifications for the study area. The fast growth scenario presented more midand high-rise buildings for the urban and town centre designated lots. The developed geospatial vertical urban growth model has the ability to rapidly generate larger numbers of buildings governed by the suitability map and procedural rules.
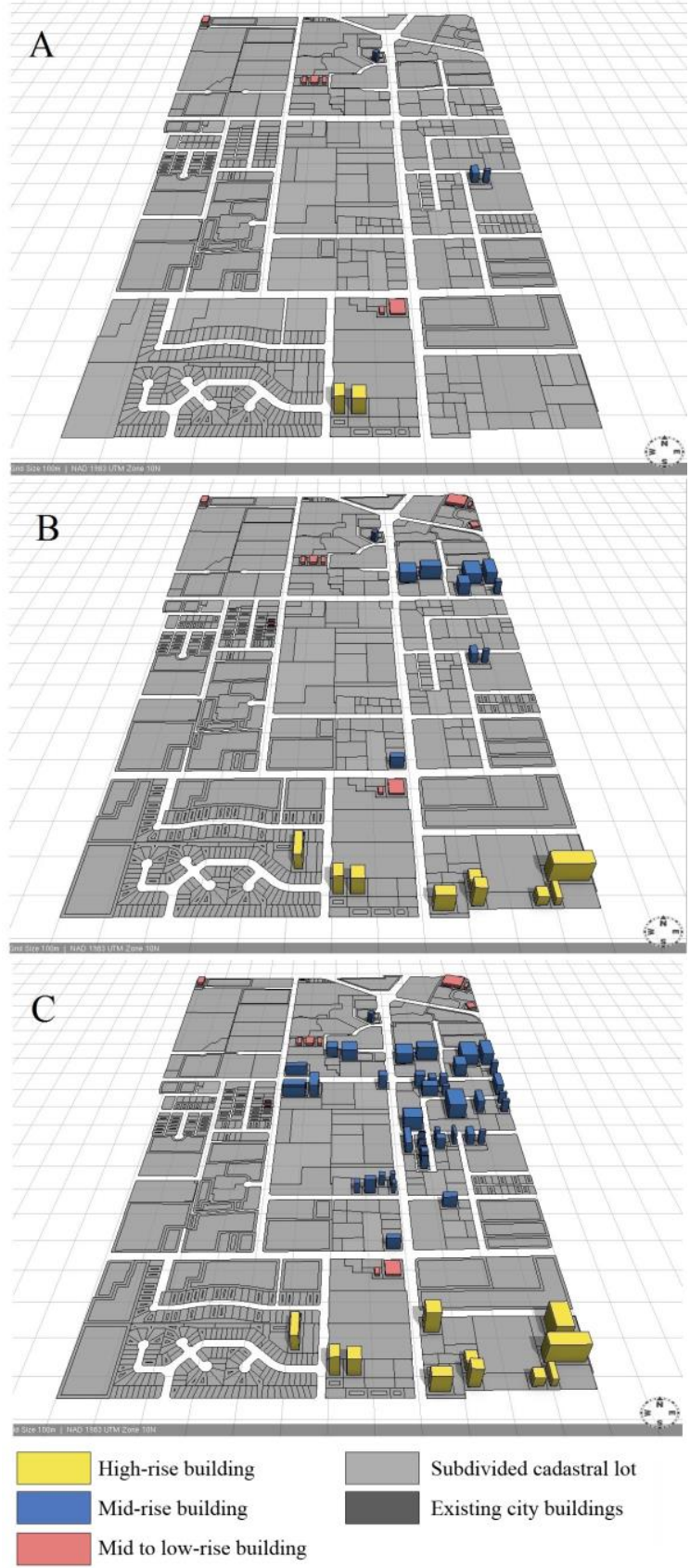

Figure 3. Modelling results for the vertical urban development obtained for three scenarios (a) slow (b) medium and (c) fast 3D growth 
This proposed geospatial modelling approach can be used by city planners to design and visualize various urban scenarios. Future work can incorporate more detailed building object designs, a temporal component, and other 3D development scenarios.

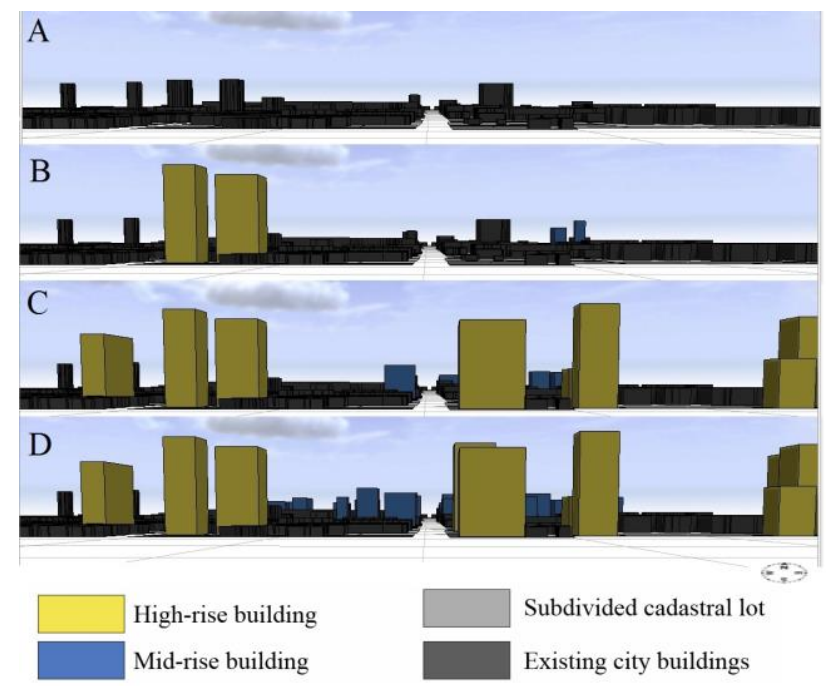

Figure 4. Visualization of the urban landscape (a) under current state, and as simulated for (b) slow (c) medium and (d) fast vertical growth development scenarios

\section{ACKNOWLEDGEMENTS}

This study was fully funded by the Natural Sciences and Engineering Research Council (NSERC) of Canada Discovery Grant awarded to Dr. S. Dragićević.

\section{REFERENCES}

Batty, M., Xie, Y., \& Sun, Z., 1999. Modelling urban dynamics through GIS-based cellular automata. Computers, Environment and Urban Systems, 23(3), 205-233.

Benguigui, L., Czamanski, D., \& Roth, R., 2008. Modelling cities in 3D: A cellular automaton approach. Environment and Planning B: Planning and Design, 35(3), 413-430.

Burton, E., 2002. Measuring urban compactness in UK towns and cities. Environment and Planning B, 29(2), 219-250.

Carver, S. J., 1991. Integrating multi-criteria evaluation with geographical information systems. International Journal of Geographical Information System, 5(3), 321-339.

Chen, G., Esch, G., Wonka, P., Müller, P., \& Zhang, E., 2008. Interactive procedural street modelling. Paper presented at the ACM Transactions on Graphics (TOG), 27(3) 103.

City of Surrey, 2014. Official Community Plan Bylaw No 18020, amended April 27, 2015. Planning and Development, Surrey, Canada. Retrieved from: http://www.surrey.ca/ City of Surrey, 2016a. Surrey Facts. Retrieved from http://www.surrey.ca

City of Surrey, 2016b. Surrey Open Data. Retrieved from: http://www.surrey.ca/city-services/

ESRI, 2013. ArcGIS Desktop version 10.2 Redlands, CA.

ESRI, 2014. CityEngine Desktop version 2014.0 Redlands, CA.
Frenkel, A., 2007. Spatial distribution of high-rise buildings within urban areas: The case of the Tel-Aviv metropolitan region. Urban Studies, 44(10), 1973-1996.

Halatsch, J., Kunze, A., \& Schmitt, G., 2008. Using shape grammars for master planning. Design computing and cognition '08 (pp. 655-673) Springer.

Lin, J., Huang, B., Chen, M., \& Huang, Z., 2014. Modelling urban vertical growth using cellular automata-Guangzhou as a case study. Applied Geography, 53, 172-186.

Malczewski, J., 2004. GIS-based land-use suitability analysis: A critical overview. Progress in Planning, 62(1), 3-65.

Mubareka, S., Koomen, E., Estreguil, C., \& Lavalle, C., 2011. Development of a composite index of urban compactness for land use modelling applications. Landscape and Urban Planning, 103(3), 303-317.

Parish, Y. I., \& Müller, P., 2001. Procedural modelling of cities. Paper presented at the Proceedings of the 28th Annual Conference on Computer Graphics and Interactive Techniques, 301-308.

Salvati, L., Zitti, M., \& Sateriano, A., 2013. Changes in city vertical profile as an indicator of sprawl: Evidence from a Mediterranean urban region. Habitat International, 38, 119125 .

Sani Roychansyah, M., Ishizaka, K., \& Omi, T., 2005. Considerations of regional characteristics for delivering city compactness: Case of studies of cities in the greater Tokyo area and Tohoku region, japan. Journal of Asian Architecture and Building Engineering, 4(2), 339-346.

Statistics Canada, 2011. CHASS Data Centre, Canadian Census Analyzer. Faculty of Arts \& Sciences, University of Toronto. Retrieved September 14, 2015, from http://datacentre.chass.utoronto.ca.proxy.lib.sfu.ca/census/ Stevens, D., Dragicevic, S., \& Rothley, K., 2007. iCity: A GISCA modelling tool for urban planning and decision making. Environmental Modelling \& Software, 22(6), 761-773.

Tamošaitienè, J., Gaudutis, E., \& Kračka, M., 2013. Integrated model for assessment of high-rise building locations. Procedia Engineering, 57, 1151-1155.

Turskis, Z., Zavadskas, E. K., \& Zagorskas, J., 2006. Sustainable city compactness evaluation on the basis of GIS and Bayes rule. International Journal of Strategic Property Management, 10(3), 185-207.

Voogd, H., 1983. Multicriteria evaluation for urban and regional planning. Taylor \& Francis.

Wang, R., 2013. 3D building modelling using images and LiDAR: A review. International Journal of Image and Data Fusion, 4(4), 273-292.

White, R., \& Engelen, G., 1993. Cellular automata and fractal urban form: A cellular modelling approach to the evolution of urban land-use patterns. Environment and Planning A, 25(8), 1175-1199. 